\title{
La educación alimentaria y nutricional desde el proceso de enseñanza-aprendizaje de la Anatomía y Fisiología Humana
}

Food and nutrition education from the teaching-learning process of Human Anatomy and Physiology

Adriel Luis Lima Rodríguez. ${ }^{1}$, Amado Lorenzo Hernández Barrenechea. ${ }^{2}$, Luis Ernesto Martínez González. ${ }^{3} \&$ Yany Leidys Laguardia Alfonso. ${ }^{4}$

\begin{abstract}
.
The recognition of the direct link between many diseases that humans currently suffer from, with their eating habits and customs, constitutes an aspect of vital importance in maintaining health. The school becomes an ideal setting to contribute to food and nutrition education from the teaching-learning process. The

\section{Resumen.}

El reconocimiento de la vinculación directa de muchas enfermedades que padece el ser humano en la actualidad, con sus hábitos $\mathrm{y}$ costumbres alimentarias, constituye un aspecto de vital importancia en el mantenimiento de la salud. La escuela se convierte en un escenario idóneo para contribuir a la

1 Universidad de Matanzas. Facultad de Educación. Cuba. adriel2905@nauta.cu, https://orcid.org/0000-0002-7353-0227

2 Universidad de Matanzas. Facultad de Educación. Cuba. amadolorenzohb@gmail.com, https://orcid.org/0000-0003-4085-8215

3 Universidad de Matanzas. Facultad de Educación. Cuba. luisernesto.martinez@umcc.cu, https://orcid.org/0000-0002-8690-8735

4 Universidad de Matanzas. Facultad de Educación. Cuba. yanisleydi.laguardia@umcc.cu, https://orcid.org/0000-0003-2234-0949
\end{abstract}


teaching-learning process of the educación alimentaria y nutricional desde discipline Human Anatomy and el proceso de enseñanza-aprendizaje. El Physiology in the Bachelor of Education proceso de enseñanza-aprendizaje de la career. Biology has the potential to disciplina Anatomía y Fisiología contribute to food and nutritional Humana en la carrera Licenciatura en education in the training of biology Educación. Biología posee teachers. The objective is to describe the potentialities of the teaching-learning process of the Human Anatomy and Physiology discipline to contribute to food and nutritional education in the training of Biology teachers. Among the methods of science used are the historical-logical, the analyticalsynthetic, the inductive-deductive and the study of documents.

Keywords: Food and nutrition education; Human Anatomy and Physiology; Teaching-learning process. potencialidades para contribuir a la educación alimentaria y nutricional en la formación del docente de biología. El objetivo es describir las potencialidades del proceso de enseñanza-aprendizaje de la disciplina Anatomía y Fisiología Humana para contribuir a la educación alimentaria y nutricional en la formación del docente de Biología. Dentro de los métodos de la ciencia utilizados se encuentran el histórico-lógico, el analítico- sintético, el inductivodeductivo y el estudio de documentos.

Palabras claves: Educación alimentaria y nutricional; Anatomía y Fisiología Humana; Proceso de enseñanzaaprendizaje.

\section{Introducción.}

En nuestro planeta una de cada tres personas está malnutrida. Una de las patologías más comunes es la obesidad, cada año mata a tantas personas como el hambre. Anualmente más de 2.6 millones de personas mueren debido a esta epidemia, que se centra en el exceso de grasa corporal debido al desequilibrio energético ocasionado por una alta ingesta de energía superpuesta a un bajo gasto (FAO, 2019).

Las enfermedades nutricionales están muy asociadas a los hábitos alimentarios de ahí la importancia de la educación alimentaria y nutricional de la población mundial, es conocido que dentro de las causas agravantes se encuentra el exceso o la disminución del consumo de alimentos, y no se trata solo del exceso, el déficit o acceso a los alimentos, sino de la selección correcta según las necesidades nutricionales (OMS, 2019). La escuela es el escenario idóneo para influir de manera gradual y sistemática en la educación alimentaria y nutricional de los niños, adolescentes y jóvenes, no solo por lo que aporta a la formación individual de estos, sino porque actuarán como vehículo hacia la familia, ya 
que transmitirán los conocimientos y conductas aprendidas en la escuela. La universidad constituye uno de los ambientes que se ha considerado adecuado para el desarrollo de actividades de promoción de la salud realizando actividades que permitan mejorar los conocimientos, las prácticas y las actitudes positivas hacia la educación alimentaria y nutricional (Reyes y Oyola, 2020)

La formación del licenciado en educación. Biología se desenvuelve en un contexto que implica trascendentes desafíos profesionales, expresados en la mejora continua del proceso de enseñanza-aprendizaje. En correspondencia, los planes de estudio han evolucionado en la búsqueda de mayor pertinencia para lograr los objetivos propuestos y de esta manera ser capaces de llevar hacia delante el desarrollo social, científico y tecnológico que exige el mundo contemporáneo. Dentro de los objetivos generales de esta carrera se refleja uno que establece nexos directamente relacionados con la educación para la salud y dentro de ella la educación alimentaria y nutricional: Contribuir mediante el proceso de enseñanza-aprendizaje de los contenidos relacionados con la Biología del nivel educativo de Secundaria Básica y Preuniversitario a la preparación de los alumnos para la vida, a la cultura científica, a su formación política e ideológica y a su educación en valores, bioético, ambiental y para la salud (MES, 2016).

El proceso de enseñanza-aprendizaje del sistema de conocimientos de la Anatomía y Fisiología Humana como ciencia morfológica y fisiología de la biología, tiene un papel importante en la educación para la salud y dentro de ella en la educación alimentaria y nutricional de la nueva generación de docentes de biología, ya que brinda los conocimientos esenciales acerca de los tejidos, órganos, sistemas de órganos y su funcionamiento, como evidencia de la integridad bilógica existente en el organismo humano. En la formación del licenciado en educación. Biología, esta problemática constituye una prioridad, pues los futuros docentes deben fomentar una educación alimentaria y nutricional que se refleje en estilos de vida más sanos y contribuya a su formación profesional pedagógica.

El objetivo es describir las potencialidades del proceso de enseñanza-aprendizaje de la disciplina Anatomía y Fisiología Humana para contribuir a la educación alimentaria y nutricional en la formación del docente de Biología.

\section{Metodologia.}

La investigación realizada responde al paradigma cualitativo, dentro de los métodos del nivel teórico empleados se encuentra el histórico-lógico, el analítico-sintético y el inductivo-deductivo que permitieron el estudio, análisis y determinación de los antecedentes fundamentales de la educación alimentaria y nutricional desde el proceso de enseñanza-aprendizaje de la disciplina Anatomía y Fisiología Humana en la formación del docente de Biología. 


\section{Resultados.}

En el Plan de Estudios E la formación del licenciado en educación. Biología expresa que desde las disciplinas y asignaturas se pondera un carácter interdisciplinario y transdiciplinario, de manera que se eliminen las fronteras entre los contenidos de las disciplinas, al integrar los nodos interdisciplinarios y los contenidos de las asignaturas en los diferentes años académicos, lo cual contribuirá a la formación integral del futuro egresado, con un alto nivel científico. La contextualización del currículo posibilita emprender con objetividad acciones que preparen al docente de Biología en formación para desarrollar la labor instructiva y educativa con sus estudiantes y lograr una adecuada educación alimentaria y nutricional (MES, 2016).

Las disciplinas biológicas contribuyen a la formación de la concepción científica del mundo, al profundizar en el estudio del mundo vivo, y sus relaciones en la naturaleza. Además, sus contenidos propician el trabajo con temas muy importantes, tales como: la fisiología de los organismos y la relación estructura-función-funcionamiento; la educación para la salud y la sexualidad responsable, la educación ambiental, de esta manera se propicia la educación para la salud de forma interdisciplinaria (MES, 2016). Desde las disciplinas biológicas se le debe dar salida a las estrategias curriculares, ya que constituyen un aspecto de gran importancia a tener en cuenta durante el proceso de enseñanza-aprendizaje, es por ello que se indica un trabajo intencionado, planificado y organizado desde los contenidos de las asignaturas. El cumplimiento de la estrategia curricular "Educación para la salud y la sexualidad con enfoque de género" debe contribuir de forma efectiva a la educación alimentaria y nutricional de los docentes en formación (Comisión Nacional de Carrera, 2016)

La educación alimentaria y nutricional corresponde a todas las disciplinas de la carrera y fundamentalmente a las biológicas, o sea, las que tratan de forma directa los contenidos de la biología y su sistema de ciencias. Estas poseen la responsabilidad de entrenar y educar a los futuros licenciados en educación. Biología para valerse con sus propios conocimientos, y aplicarlos en su vida cotidiana con el objetivo de mantener una elevada calidad de vida y educar a la nueva generación de cubanos. Esto solo se logra en la formación profesional pedagógica con la actuación protagónica en la ejecución de acciones que fortalecen la confianza en las potencialidades individuales.

La disciplina Anatomía y Fisiología Humana forma parte de las disciplinas biológicas del currículo propio de la carrera Licenciatura en Educación. Biología. Ha sido diseñada para cumplir con los requerimientos del nuevo Plan de Estudios E y se debe desarrollar una vez que los estudiantes hayan concluido la disciplina Zoología. Constituye una disciplina importante para un numeroso grupo de profesionales. En el caso de los docentes de Bilogía, su dominio se hace necesario por cuanto, en lo fundamental, aporta las herramientas necesarias para poder desarrollar los programas de noveno, décimo y onceno grados de la educación general media, a la vez que, por su relación con otras disciplinas, permite explicar procesos o fenómenos de estas. 
El estudio de esta disciplina se hace importante, además, porque dota a los futuros profesores de las vías para explicar la relación estructura-función que se da entre los diferentes tejidos, órganos y sistemas de órganos del cuerpo humano. Su conocimiento es de gran valor para poder explicar el programa de Educación Sexual y para la Salud. La integración en el proceso de estudio contribuye al cumplimiento de los objetivos generales de esta disciplina, en particular del que plantea: "Aplicar consecuentemente el Programa de Educación para la Salud y Sexual, como base para su cumplimiento en su futura labor docente" (Lezcano \& Martínez, 2016, pág. 2).

El sistema de conocimientos que es abordado en la disciplina Anatomía y Fisiología Humana en la formación del licenciado en educación. Biología, cuenta con las posibilidades reales de tratar con claridad y relevancia diversos temas sobre educación alimentaria y nutricional. Estos contenidos por su esencia permiten hacer alusión en las clases a los contenidos esenciales que se declaran en el documento normativo La Promoción y Educación para la Salud como parte del perfeccionamiento del Sistema Nacional de Educación reflejados en el área de contenido de la educación alimentaria y nutricional. En correspondencia con el análisis del programa de la asignatura, se precisan tópicos para contribuir a la educación alimentaria y nutricional:

Integridad y relaciones del organismo como un todo y con el medio ambiente que le rodea. Importancia de la higiene individual y colectiva. Conceptos de salud. Papilas gustativas: situación y características generales. Importancia funcional. Meningoencefalitis. Aspectos anatómicos y producción hormonal del páncreas endocrino. Control de la secreción de la insulina y el glucagón y sus efectos fisiológicos. Salud y medidas higiénicas. Enfermedades más comunes y su incidencia en los casos de discapacidades físicas y/o mentales. Sistema digestivo. Conceptos de nutrientes, alimento, alimentación, nutrición y dieta balanceada. Masticación; estructuras que intervienen e importancia. Hidrólisis de proteínas y grasas emulsionadas. Quimo gástrico. Páncreas e hígado. Jugo pancreático y bilis: composición e importancia en la digestión intestinal. Mecanismo de absorción de los productos finales de la digestión (incluir vitaminas, minerales y agua). Salud y medidas higiénicas. Algunas enfermedades más comunes y su coincidencia en casos de desviaciones físicas y/o mentales. Sistema circulatorio. Salud y medidas higiénicas. Algunas enfermedades más comunes y su coincidencia en casos de desviaciones físicas y/o mentales. Sistema renal. Salud y medidas higiénicas. Integración de las funciones vegetativas en el funcionamiento del organismo. Importancia. Relación entre los sistemas de regulación y las funciones vegetativas. Ejemplos. Salud y medidas higiénicas (Lezcano \& Martínez, 2016, pág. 3).

En consecuencia, el proceso de educación alimentaria y nutricional desde el proceso de enseñanza-aprendizaje de la disciplina Anatomía y Fisiología Humana en la formación del licenciado en educación. Biología, debe integrarse a diversos tipos de actividades que tengan carácter instructivo, desarrollador y formativo. Existe una unidad dialéctica entre la formación y el desarrollo. De manera que la educación debe considerarse en su función desarrolladora y formativa. Sobre esta base, se debe tener en cuenta el carácter subjetivo de las influencias educativas en el proceso de formación de la personalidad para garantizar 
la formación y el desarrollo de los estudiantes. También resulta importante considerar la actuación de estos como un indicador fundamental para valorar los resultados en el proceso de formación. La formación y desarrollo de cada sujeto tiene lugar en el proceso de educación de la personalidad y ocurre en dependencia de las características individuales de cada uno de ellos (Vilaplana, 2011).

El proceso de educación alimentaria y nutricional desde el proceso de enseñanzaaprendizaje de la disciplina Anatomía y Fisiología Humana en la formación del licenciado en educación. Biología es consecuente con los postulados del enfoque histórico-cultural de L. S. Vigotski (1896-1934) y sus seguidores. En este caso el autor ha considerado los aportes relacionados con el carácter dinámico de la psiquis humana; el carácter mediatizado de los procesos psíquicos; los estadios del desarrollo; la relación de la educación y el desarrollo y la unidad entre lo cognitivo y lo afectivo (Morenza, 2010).

Al considerar el carácter dinámico de la psiquis, se parte de reconocerla como una propiedad del ser humano como ser material y a su vez como un producto social, consecuencia del desarrollo histórico de la humanidad. El accionar pedagógico desempeña un papel fundamental en el desarrollo individual de la psiquis de los estudiantes, al crear condiciones específicas para la educación alimentaria y nutricional desde el proceso de enseñanza-aprendizaje de la disciplina Anatomía y Fisiología Humana en la formación del licenciado en educación. Biología. Es por ello que se tiene en cuenta el carácter social de la psiquis al responder a las características individuales y necesidades de este grupo.

Por otra parte, el carácter mediatizado de los procesos psíquicos se manifiesta al satisfacer los aprendizajes necesarios que en este sentido demandan los estudiantes. De esta manera, se implementa “....un proceso de aprendizaje donde está implícita la actividad y la comunicación como instrumentos mediadores de la actividad psíquica en el proceso de educación nutricional" (Ministerios del Interior, de Educación y Cultura y de Sanidad y Consumo, 2015, pág. 4).

Desde el punto de vista pedagógico, el plano intrapsicológico representa lo que el estudiante es capaz de hacer por sí mismo, en tanto el interpsicológico se refiere al espacio en el cual realiza las tareas con ayuda de otros, que de alguna manera las dominan mejor que él. De ello resulta necesario admitir que la educación alimentaria y nutricional desde el proceso de enseñanza-aprendizaje de la disciplina Anatomía y Fisiología Humana es un proceso que tiene un doble carácter: en lo individual y en lo social; donde la actividad y la comunicación entre los estudiantes es un elemento de significativa importancia, de ahí que siempre sea dependiente del contexto social en que tenga lugar.

En el enfoque histórico cultural, la situación social del desarrollo (SSD) es otro concepto que permite analizar la particular correlación entre educación y desarrollo. Este sostiene que, en cada estudiante, según sus experiencias, la situación social del desarrollo es diferente, es propia de cada individuo en particular. De ahí que en este proceso se tiene en cuenta el respeto a la individualidad del estudiante. En este contexto la concepción 
vigotskiana acerca de que la educación y la enseñanza conducen y guían el desarrollo; se expresa de forma clara en los conceptos Zona de Desarrollo Próximo (ZDP) y Zona de Desarrollo Actual (ZDA). En este caso la ZDA coincide con el nivel de conocimientos y habilidades que presentan los estudiantes que permite valorar sus correctos hábitos nutricionales y alimentarios y la contribución del proceso de enseñanza-aprendizaje de la disciplina Anatomía y Fisiología Humana para la educación alimentaria y nutricional.

Durante el desarrollo de la personalidad se complementan los aspectos cognoscitivos y afectivos, así como su expresión en la conducta, como resultado de la unidad de lo cognitivo y lo afectivo como expresión del carácter integral del psiquismo humano. En consecuencia, las acciones que se ejecuten para contribuir a la educación alimentaria y nutricional desde el proceso de enseñanza-aprendizaje de la disciplina Anatomía y Fisiología Humana en la formación del licenciado en educación. Biología, deben potenciar el desarrollo cognitivo y afectivo de los estudiantes, como resultado de la concreción del principio de la unidad de lo instructivo, lo educativo y lo desarrollador en el proceso de educación de la personalidad (Castellanos, Castellanos, Llivina, \& Silverio, 2002)

El encargo social asignado a la universidad, y en particular en las carreras pedagógicas para la formación integral de los estudiantes, resulta de gran utilidad e importancia en su contribución a la educación alimentaria y nutricional de estudiantes de la Licenciatura en Educación. Biología, sobre la base del proceso de enseñanza-aprendizaje de la disciplina Anatomía y Fisiología Humana, dado en lo fundamental por la dimensión de este aspecto en el cumplimiento de los objetivos en este nivel de educación. Los autores asumen los aspectos relacionados con la educación y la enseñanza como conductoras del desarrollo, y el papel del medio en este sentido para contribuir a la educación alimentaria y nutricional. Corresponde entonces, en particular, al profesor de Biología la misión de dirigir el desarrollo de cada estudiante, según sus características individuales y sus necesidades y así contribuirá a su educación alimentaria y nutricional. A su vez, la comunicación con sus coetáneos es muy importante y las presiones del grupo juegan un papel significativo, este aspecto debe ser aprovechado por los docentes de forma óptima.

De esta manera se contribuirá a la formación de conocimientos, actitudes, valores y modos de comportamientos que les permita aprender a decidir por sí mismos, reconocer qué es lo factible y positivo, no solo para él, sino también para sus compañeros. En esa relación del individuo con sus semejantes, en su actuación, así como mediante la actividad y la comunicación, es que se forma y desarrolla su personalidad. Al considerar que "Dado que la personalidad está sujeta a un condicionamiento histórico-social y dialéctico, caracterizado por el carácter activo del sujeto..." (González \& Lopez, 2010, pág. 9). No se puede esperar que todos los estudiantes se apropien de hábitos nutricionales y alimentarios de la misma manera. Esto justifica la atención a la diversidad para contribuir a la educación alimentaria y nutricional de los estudiantes.

Favorecer en los estudiantes la apropiación del sistema de conocimientos de la Anatomía y Fisiología Humana relacionados con la educación alimentaria y nutricional hace posible 
el desarrollo de habilidades generales y específicas que posibilitan su actuación en correspondencia con su educación alimentaria y nutricional, la formación de nuevas habilidades y/o el desarrollo de las que ya poseía el estudiante. Este aspecto resulta fundamental para garantizar el vínculo con la vida, con el doble propósito de que el estudiante aprenda más a partir de su realidad y esté en mejores condiciones de solucionar problemas de la vida cotidiana.

\section{Conclusiones.}

- El desarrollo de una correcta educación alimentaria y nutricional, no puede considerarse de ninguna manera tarea sencilla, ya que implica lograr transformaciones en los hábitos alimentarios. Es necesario modificar maneras de pensar y actuar relacionados con la selección de los alimentos. Es un aspecto necesario en las condiciones del mundo actual, para lo cual existen orientaciones y precisiones que deben ser cumplimentadas en la labor pedagógica de los educadores del país, donde los profesores de Biología tienen una alta responsabilidad.

- La educación alimentaria y nutricional se encuentra insertada al proceso de enseñanza-aprendizaje de los contenidos de las disciplinas biológicas en la formación del licenciado en educación. Biología, al favorecer la adquisición de los conocimientos, hábitos y habilidades necesarios, con la finalidad de contribuir a manifestar una educación alimentaria y nutricional adecuada y educar a la nueva generación de estudiantes.

- El proceso de enseñanza-aprendizaje de contenidos de la disciplina Anatomía y Fisiología Humana está dirigido a contribuir a la educación para la salud y dentro de ella a la educación alimentaria y nutricional de los estudiantes. Debe caracterizarse por propiciar la activación-regulación, la significatividad y la motivación, para ello, las situaciones de enseñanza y de aprendizaje que se desarrollen deben favorecer el aprendizaje productivo, creativo y crítico, así como las reflexiones de los estudiantes sobre su aprendizaje.

\section{Referencias bibliográficas.}

Castellanos, D., Castellanos, B., Llivina, J., \& Silverio, M. (2002). Hacia una concepción del aprendizaje desarrollador. La Habana: Pueblo y Educación.

Comisión Nacional de Carrera. (2016). Indicaciones Metodológicas y de Organización de la carrera Licenciatura en Educación. Biología. La Habana: Universidad de Ciencias Pedagógicas Enrique José Varona.

Gómez, Y., López, A. B., Torres, M. A., Carvajal, C., García, I., Roca, A. A., \& Mazón, Y. (2015). La Promoción y Educación para la Salud como parte del perfeccionamiento del Sistema Nacional de Educación. La Habana: MINED. 
González, L., \& Lopez, G. (10 de Junio de 2010). La comunicación educativa en el aula: una alternativa para la enseñanza de las teorías de la comunicación. Obtenido de Dianet (80): http:// dialnet.unirioja.es

Lezcano, E. L., \& Martínez, L. E. (2016). Progra de desiciplina de Anatomía y Fisiología Huamanas. La Habana: MES.

Ministerios del Interior, de Educación y Cultura y de Sanidad y Consumo. (26 de 03 de 2015). Marco del Convenio de Colaboración para fomentar la educación para la salud en la escuela. Recuperado el 11 de 12 de 2016, de Guía. Nutrición saludable y prevención de los trastornos alimentarios: Disponible en: http://www.aeesme.org/wp-content/uploads/docs/guia_nutricion_saludable.pdf

Morenza, L. (2010). Pre-Reunión. Paradigmas contemporáneos de aprendizaje de L. S. Vigostki y Piaget al procesamiento de la información. La Habana: Universidad de La Habana.

Organización de las Naciones Unidas para la Alimentación y la Agricultura. (2019). El estado de la Seguridad Alimentaria y Nutricional en el mundo. Roma: FAO.

Organización Mundial de la Salud. (2019). Informe de la Nutrición Mundial 2019. Suiza: OMS.

Reyes, S. E., \& Oyola, M. S. (2020). Programa educativo nuricional en estudiantes universitarios. Iberoamericana de las Ciencias de la Salud 9(17), 12-33.

Vilaplana, M. (2011). Educación nutricional en el niño y el adolescente. Ámbito farmaceútico. Nutrición 30(3), 43-50.

\section{\Ciencia}




\section{PARA CITAR EL ARTÍCULO INDEXADO.}

Lima Rodríguez, A. L., Hernández Barrenechea, A. L., Martínez González, L. E., \& Laguardia Alfonso, Y. L. (2021). La educación alimentaria y nutricional desde el proceso de enseñanza-aprendizaje de la Anatomía y Fisiología Humana . Anatomía Digital, 4(2), 30-39. https://doi.org/10.33262/anatomiadigital.v4i2.1615

\section{Ciencia \\ LDigital}

El artículo que se publica es de exclusiva responsabilidad de los autores y no necesariamente reflejan el pensamiento de la Revista Anatomía Digital.

El artículo queda en propiedad de la revista y, por tanto, su publicación parcial y/o total en otro medio tiene que ser autorizado por el director de la Revista Anatomía Digital.
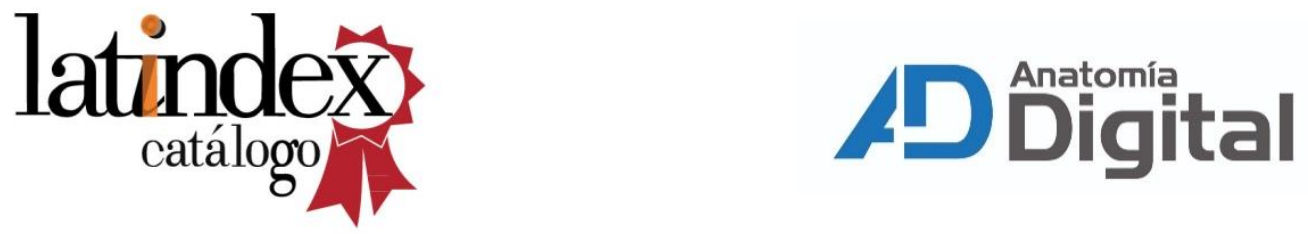